\title{
Shortest link scheduling in wireless networks under the Rayleigh fading model
}

\author{
Baogui Huang ${ }^{1} \mathbb{B}$, Jiguo $\mathrm{Yu}^{2,3,4^{*}}$, Chunmei Ma${ }^{1}$, Fengyin $\mathrm{Li}^{1}$ and Guangshun $\mathrm{Li}^{1}$
}

\section{${ }^{*}$ Correspondence:}

jiguoyu@sina.com

${ }^{4}$ Shandong Provincial Key

Laboratory of Computer

Networks, Jinan, China

Full list of author information

is available at the end of the

article

\begin{abstract}
Many shortest link scheduling algorithms adopt non-fading SINR interference model, which assumes that the received signal power will always remain determinate as long as the transmission power of the corresponding sender is fixed. In fact, because environment always influences the propagation of radio signals, the received signal power is by no means a certain value. Rayleigh fading is a statistical model for radio signals propagation. It assumes that the strength of a signal on a receiver is a random variable, varying with the Rayleigh distribution. This paper proposes a shortest link scheduling algorithm under the Rayleigh fading model (SLSRF). The SLSRF partitions the wireless network area into hexagons and colors the hexagons with three different colors such that two neighboring hexagons have different colors. The senders of the links scheduled simultaneously are arranged in hexagons with the same color. The correctness of the SLSRF is proved through theoretical analysis, and the efficiency is illustrated by elaborate simulations. Our simulation results demonstrate that the schedule delay of SLSRF is less than that of some results under the non-fading SINR interference model. Furthermore, we extend the SLSRF to a distributed version, which is suitable for large wireless networks.
\end{abstract}

Keywords: Wireless network, Link scheduling, Rayleigh fading, SINR

\section{Introduction}

The fifth-generation (5G) wireless systems provide support for many applications, such as smart home, smart city [1,2], smart traffic, smart health care [3], industry 4.0 [4], smart agriculture, unmanned aerial vehicles [5-11] and so on. Many special applications require ultrareliable low-latency communications (URLLC) for $5 \mathrm{G}$ systems $[12,13]$. The strict requirements of URLLC have led to research in many fields, including link scheduling problems for $5 \mathrm{G}$ systems (e.g., [14-16]). Additionally, the link scheduling problems are closely related to the fundamental issues in 5G wireless networks such as coverage, connectivity, capacity and throughput, and delay. The correctness of any link scheduling algorithm relies on the underlying interference model. Moscibroda and Wattenhofer [17] shifted interference models from simple graph-based models to a more realistic model, that is, the signal-to-interference plus noise ratio (SINR) model. Recently, much research on link scheduling problems has been conducted under the SINR model (e.g., [18-25]). The SINR model considers global interference, i.e., the sum of all interfering

(c) The Author(s) 2021. This article is licensed under a Creative Commons Attribution 4.0 International License, which permits use, sharing, adaptation, distribution and reproduction in any medium or format, as long as you give appropriate credit to the original author(s) and the source, provide a link to the Creative Commons licence, and indicate if changes were made. The images or other third party material in this article are included in the article's Creative Commons licence, unless indicated otherwise in a credit line to the material. If material is not included in the article's Creative Commons licence and your intended use is not permitted by statutory regulation or exceeds the permitted use, you will need to obtain permission directly from the copyright holder. To view a copy of this licence, visit http://creativecommons. org/licenses/by/4.0/. 
signals plus the background noise. Therefore, the SINR model closely covers the main features of complex fading models such as the two-ray-ground model without losing too much of the simplicity needed for algorithmic design. In general, the path loss exponential model is taken into account when one designs link scheduling algorithms. That model assumes any signal transmitted with power $P_{t}$ is always received after distance $d$ with power $P_{t} / d^{\alpha}$, where $\alpha$ is the path loss exponent and $2<\alpha<6$. However, the path loss exponential model does not take short-term fluctuations into account, such as fading. In fact, the radio propagation model is very complex, and the power of the received signal is by no means a determinate value. It is an empirical mathematical formulation for the characterization of radio wave propagation as a function of frequency, distance and other conditions. Experimental work conducted in Manhattan [26] found that Rayleigh fading can be a useful model in heavily built-up city centers where there is no line of sight between the transmitter and receiver and many buildings and other objects attenuate, reflect, refract, and diffract the signals. In recent years, the Rayleigh fading model has been considered in numerous research works, such as [27-31].

In this paper, we focus on the shortest link scheduling problem under the Rayleigh fading model. Given a set of $m$ links $E=\left\{e_{1}, e_{2}, \ldots, e_{m}\right\}$, the shortest link scheduling algorithms partition $E$ into subsets $E_{1}, E_{2}, \ldots, E_{K}$ such that $K$ is minimal and the successful transmission of all links in each subset satisfies the Rayleigh fading model and the SINR interference constraint.

The main contributions can be summarized as follows.

- We consider the Rayleigh fading model, take the accumulated interference into account and design an efficient link scheduling algorithm, named the shortest link scheduling algorithm under the Rayleigh Fading (SLSRF) model. Under the Rayleigh fading model, the received power at the receiver is a random variable, not a determinate value. Therefore, it is more difficult to design link scheduling algorithms under the Rayleigh fading channel model than under the path loss exponential model. In addition, we take the SINR interference model into account, and transform the global interference into local interference skillfully by partitioning the network area into hexagons. Each sender is located in a hexagon, and at most one link is selected from a hexagon such that the interference of every two links is upper-bounded. Theoretical analysis proves that the interference of a link, i.e., $e_{1}$, which is beyond some distance to a link, i.e., $e_{2}$, does not affect the Rayleigh success probability of $e_{2}$.

- The SLSRF simultaneously selects one link from each hexagon with the same color, and this selection process is executed one by one. In fact, the selection process can execute concurrently and does not lead to conflicts. This situation motivates us to propose a distributed link scheduling algorithm, which is a distributed version of the SLSRF; thus, we refer to it as the DI-SLSRF. We propose a leader election algorithm, and senders residing in the same hexagon execute the leader election subroutine with a distributed pattern.

- We validate the correctness and performance of the SLSRF and DI-SLSRF by elaborate simulations. Although the ideas behind the SLSRF and DI-SLSRF are similar to those behind the SLSPC and SLSUM [22], SLSRF and DI-SLSRF get less schedule delay than that of SLSPC and SLSUM. 


\section{Method and organization of the paper}

This work is organized in three main steps.

- First, we did an extensive study of the state of the art on link scheduling problems, radio propagation model and interference model. We found that the link scheduling strategy based on plane partition can effectively control the global interference of the SINR model. Most algorithms partitioned the network region into rectangles and used the non-fading SINR model. Our research idea is to apply the regular hexagonal partition scheme to the non-fading SINR model link scheduling. In Sect. 3, we reviewed the related work. And, the models and assumptions were described in Sect. 4.

- Then, we proposed a shortest link scheduling algorithm based on the regular hexagonal partition under the Rayleigh fading model, named SLSRF. Communication links were grouped according to the distance between the senders and their corresponding receivers. For each group, we partitioned network area into hexagons and colored them such that two neighboring hexagons had different colors. Time is partitioned into slots; at each slot, we scheduled at most one link from each hexagon having the same color. We outlined the SLSRF, proved its correctness and effectiveness by theoretical analysis and elaborate simulations in Sects. 5 and 6, respectively.

- Last, we proposed a leader election algorithm, named LE, and extended the SLSRF to a distributed version in Sect. 7. Before sending a message, a sender must compete for the channel by executing the LE and the leader can send a message to its corresponding receiver. We concluded this paper in Sect. 8.

\section{Related work}

The link scheduling problem under the SINR model originated from the work of Moscibroda and Wattenhofer [17]. From then on, much work on link scheduling has been conducted under the SINR model. Because of the global characteristic, link scheduling problems are NP-hard under the SINR model [32]. By partitioning the network area into some cells, global interference becomes localized, which is an effective method for designing a link scheduling algorithm.

Goussevskaia et al. [32] partitioned the network area into squares and divided the problem instance into disjoint link length classes and then constructed a feasible schedule for each length class using a greedy strategy. The interference model used in [32] is an approximation of the SINR model, in which the effect of the ambient noise is neglected. In this case, the SINR model is simplified to the signal-to-interference ratio (SIR) model, in which the transmission range of a link can be infinite. Thus, the possible number of link length classes can also be infinite.

Blough et al. [33] noted that black-gray links would affect the performance of the link scheduling algorithm. Black-gray links mean that their lengths are equal or near the maximum transmission range of the sender; these are treated separately, as they are difficult to schedule. In contrast to [32], Blough et al. [33] considered the exact 
SINR model, that is, both the ambient noise and the accumulated interference at a receiver were considered.

$\mathrm{Xu}$ et al. [34] studied the maximum weighted independent set of links (MWISL) problem under the SINR model with oblivious power assignment in wireless networks. They partitioned the network area into large squares and then partitioned each large square into small squares. Next, they utilized partition and shifting strategies to find multiple sets of well-separated links and then selected the one with the largest weight. Similar to [34], by combining the partition and shifting strategies with a pick-and-compare scheme, Zhou et al. [35] presented a class of localized scheduling algorithms with a provable throughput guarantee under the SINR constraint. Different from [34, 35] obtained a maximum independent set of links in a small square instead of choosing one link from a small square. The basic idea of the algorithms in [35] was to create a set of disjoint local link sets in which the scheduling can be done independently without violating the global interference constraints. The distance between two cells is determined by the longest link, resulting in a loose approximation factor.

Since the hypergraph model can limit the interference of links around itself, the interference from links at farther distances is negligible. Then, based on the characteristic of the hypergraph and the idea of [33], combining the SINR and hypergraph models, Wang et al. [36] proposed an improved algorithm for shortest link scheduling with oblivious power assignment. The main difference between [33, 36] is as follows. (1) [36] does not group the links into subsets; (2) [36] eliminates black-gray links by improving their transmission power; and (3) [36] chooses as many links at each time slot as possible instead of one link, as in [33]. Therefore, [36] decreased the latency of all links.

Based on the fact that a short link can tolerate large interference while a long link tolerates small interference, Huang et al. [37] proposed a novel approximation algorithm for shortest link scheduling with oblivious power control. The motivation of [37] is that if two transmission links are far from each other, the interference of one link on the other should be small under the SINR constraint. By partitioning the links into disjoint local link sets that are a certain distance away from each other, independent scheduling inside each local link set is possible. Similar to [34,37] partitioned the network area into large squares and partitioned each large square into small squares. For every small square, [37] designed a maximum link scheduling algorithm to select as many links as possible.

All the works mentioned above partitioned the network area into squares. Yu et al. [22] considered another partition strategy, that is, the hexagon partition. The algorithms of [22] were motivated by cellular networks, in which each cell uses a set of frequencies that are different from those of neighboring cells to avoid interference and provide guaranteed bandwidth. Simulations demonstrated that the hexagon partition was more efficient than the square partition employed by [32,33]. Moreover, [22] considered the energy consumption. The algorithms of [22] consume less energy than those of [32,33] in the case of approximate latency of the link set.

However, a major deficiency of the SINR model is that if the sending power is fixed, i.e., $P$, the received power at the receiver at a distance $d$ from the sender is always $P d^{-\alpha}$, that is, the SINR model ignores the influence of the multipath fading effect on the signal transmission. Therefore, when these results are applied to the actual network environment, the bound conditions are faced with great challenges. 
Liu and Haenggi [38] addressed the throughput with the Rayleigh fading channel and considered the impact of the topology on the network performance by comparing networks with a random topology and three regular topologies, including the square, triangle and hexagon topologies. Among the three regular networks (square, triangle, and hexagon), the hexagon network provides the highest throughput since every node has only three nearest neighbors, which is the smallest number of neighbors among the three networks. Dames et al. [39] examined the relationship between the nonfading SINR model and the Rayleigh fading model and found that we can apply existing algorithms for the nonfading SINR model in the Rayleigh fading scenario while losing only a factor of $O\left(\log ^{*} n\right)$ in the approximation guarantee.

\section{Models and definitions}

The topology of a wireless network is modeled as a directed graph $G=(V, E)$, where $V$ denotes the set of nodes and $E$ denotes the set of links. All nodes are deployed in a 2-dimensional plane area randomly. The nodes are partitioned into two classes: one is the set of senders, denoted by $S$, and the other is the set of receivers, denoted by $R$, that is, $V=S \cup R$, $S=\{s \mid s$ is a sender $\}$ and $R=\{r \mid r$ is a receiver $\}$. If a sender $u$ sends a signal to a receiver $v$, we use a directed edge $e$ to denote the communication link, that is, $e=(u, v)$. Assume that all links have different senders and receivers, that is, if $e_{1}=\left(u_{1}, v_{1}\right)$ and $e_{2}=\left(u_{2}, v_{2}\right)$, then $u_{1}$, $v_{1}, u_{2}$ and $v_{2}$ are all different. Let $\|u v\|$ denote the Euclidean distance between $u$ and $v$. The distance between two links, $e_{1}=\left(u_{1}, v_{1}\right)$ and $e_{2}=\left(u_{2}, v_{2}\right)$, is defined as $\left\|u_{1} v_{2}\right\|$. A sender $u$ sends signals with power $Q_{u}$, and the power at the expected receiver $v$ is $Q_{u v}$. Note that the receiver $v$ may receive another signal from another undesirable sender, say $w$, which transmits a signal simultaneously with $u$. We say that $w$ interferes with the communication of $v$, and we use $Q_{w v}$ to denote the received power at $v$. We define the transmission probability of $u$, denoted by $p_{u}$ :

$$
p_{u}= \begin{cases}1, & u \text { transmits a signal } \\ 0, & \text { otherwise }\end{cases}
$$

\subsection{SINR model}

The SINR model is also called the physical interference model, since it reflects physical reality more accurately than the graph-based interference models. In the SINR model, a signal is received successfully if and only if the signal-to-interference plus noise ratio (SINR) at the receiver is above a threshold determined by the communication hardware and the modulation and coding scheme. More formally, a communication over a link $e=(u, v)$ succeeds if and only if the following condition holds:

$$
\frac{Q_{u v}}{N+I} \geq \beta
$$

where $\beta$ denotes the minimum SINR value required for a signal to be successfully received, $N$ is the ambient noise and $I$ is the accumulated interference caused by other senders transmitting simultaneously with $u$, denoted by $I S_{u}$, that is, $I S_{u}=\{w \mid w$ is transmitting a signal simultaneously with $u$ \}. Therefore,

$$
I=\sum_{w \in I S_{u}} Q_{w v}
$$




\subsection{Rayleigh fading model}

The Rayleigh fading is a statistical model for determining the effect of a propagation environment on a radio signal. It assumes that the magnitude of a signal at a receiver is a random variable, which varies according to the Rayleigh distribution. When the sender $u$ transmits signals with power $Q_{u}$, in the Rayleigh fading model, the power received at $v$ is exponentially distributed with a mean value $\bar{Q}_{u v}$. The random variable of the received power is denoted by $q_{u v}$. Then,

$$
p_{Q_{u v}}\left(q_{u v}\right)=\bar{Q}_{u v}^{-1} \cdot e^{-q_{u v} \bar{Q}_{u v}^{-1}} .
$$

Here, $\bar{Q}_{u v}=Q_{u}\|u v\|^{-\alpha}$ denotes the average received power, with $2<\alpha<6$ denoting the path loss exponent, the exact value of which depends on the external conditions of the medium (humidity, obstacles etc.).

If two links $e_{1}=\left(u_{1}, v_{1}\right)$ and $e_{2}=\left(u_{2}, v_{2}\right)$ transmit simultaneously, the interference of $e_{1}$ on $e_{2}$ is exponentially distributed with a mean

$$
\bar{Q}_{u_{1} v_{2}}=Q_{u_{1}}\left\|u_{1} v_{2}\right\|^{-\alpha},
$$

that is,

$$
p_{Q_{u_{1} \nu_{2}}}\left(q_{u_{1} v_{2}}\right)=\bar{Q}_{u_{1} \nu_{2}}^{-1} \cdot e^{-q_{u_{1} \nu_{2}} \bar{Q}_{u_{1} \nu_{2}}^{-1}}
$$

Let $E_{t}$ denote the set of links that transmit signals in slot $t$ simultaneously; then, the accumulated interference on $e_{i} \in E_{t}$ is $I=\sum_{e_{j} \in E_{t} \wedge j \neq i} I_{e_{j} e_{i}}$. Under the SINR model and a fixed $I$, the probability that $e_{i}$ can successfully transmit the signal is

$$
\mathrm{P}\left[\frac{Q_{u_{i} \nu_{i}}}{I+N} \geq \beta \mid I=x\right] .
$$

Theorem 1 Under the condition of I, the probability that $e_{i}=\left(u_{i}, v_{i}\right)$ transmits the signal successfully is

$$
\mathrm{P}=\exp \left(-\beta N Q_{u_{i}}^{-1}\left\|u_{i} v_{i}\right\|^{\alpha}\right) \prod_{e_{j} \in E_{t} \wedge j \neq i}\left(1+\beta\left\|u_{i} v_{i}\right\|^{\alpha}\left\|u_{j} v_{i}\right\|^{-\alpha}\right)^{-1} .
$$

Proof Taking the expectation over $I$, we have

$$
\begin{aligned}
\mathrm{P} & {\left[\frac{Q_{u_{i} v_{i}}}{I+N} \geq \beta \mid I=x\right]=\mathrm{E}\left[\mathrm{P}\left[\frac{Q_{u_{i} v_{i}}}{I+N} \geq \beta\right]\right] } \\
& =\exp \left(-\frac{\beta N}{\bar{Q}_{u_{i} v_{i}}}\right) \mathrm{E}\left[\prod_{e_{j} \in E_{t} \wedge j \neq i} \exp \left(-\frac{\beta I_{e_{j} e_{i}}}{\bar{Q}_{u_{i} v_{i}}}\right)\right] \frac{1}{\bar{Q}_{u_{j} v_{i}}} \exp \left(-\frac{q_{u_{j} v_{i}}}{\bar{Q}_{u_{j} v_{i}}}\right) d\left(q_{u_{j} v_{i}}\right) \\
& =\exp \left(-\frac{\beta N}{\bar{Q}_{u_{i} v_{i}}}\right) \prod_{e_{j} \in E_{t} \wedge j \neq i} \frac{1}{\bar{Q}_{u_{j} v_{i}}} \int_{0}^{\infty} \exp \left(-\left(\frac{\beta}{\bar{Q}_{u_{i} v_{i}}}+\frac{1}{\bar{Q}_{u_{j} v_{i}}}\right) q_{u_{j} v_{i}}\right) d\left(q_{u_{j} v_{i}}\right) \\
& =\exp \left(-\beta N Q_{u_{i}}^{-1}\left\|u_{i} v_{i}\right\|^{\alpha}\right) \prod_{e_{j} \in E_{t} \wedge j \neq i}\left(1+\beta\left\|u_{i} v_{i}\right\|^{\alpha}\left\|u_{j} v_{i}\right\|^{-\alpha}\right)^{-1}
\end{aligned}
$$


Here, the last equation holds only if $Q_{u_{j}}=Q_{u_{i}}$, which can be guaranteed by an assumption in the next section.

\section{Shortest link scheduling algorithm with Rayleigh fading}

Next, we outline the shortest link scheduling with Rayleigh fading (SLSRF). The SLSRF comprises three critical steps: classifying links, partitioning the network area and selecting links from hexagons.

\subsection{Classify links}

Let maxlen and minlen denote the maximum length and minimum length of the links, respectively. We classify the links into some disjoint groups according to their lengths, that is,

$$
\left\{\begin{array}{l}
E=C_{0} \cup C_{1} \cup \cdots \cup C_{K}, K=\lfloor\log (\text { maxlen } / \text { minlen })\rfloor, \\
C_{i}=\left\{e \mid 2^{i} \text { minlen } \leq\|e\|<2^{i+1} \text { minlen }\right\}, \quad i=0, \ldots, K, \\
C_{i} \cap_{0 \leq i<j \leq K} C_{j}=\emptyset .
\end{array}\right.
$$

All links in the same group have the same sending power. Let $Q_{i}=8 \beta N\left(2^{i+1} \text { minlen }\right)^{\alpha}$ denote the sending power of the $i$ th group.

\subsection{Partition the network area and select links}

For the $i$ th group, let

$$
\mu=1+\left(\beta /\left((5 / 4)^{1 / 6}-1\right)\right)^{1 / \alpha}
$$

We partition the network area into hexagons with a side length of $\mu 2^{i+1}$ minlen and color them such that no two adjacent hexagons have the same color; see Fig. 1. We use 1, 2 and 3 to denote three different colors. A link belongs to a hexagon if its sender resides in the hexagon. Next, we consider the hexagons group by group and select the links for a

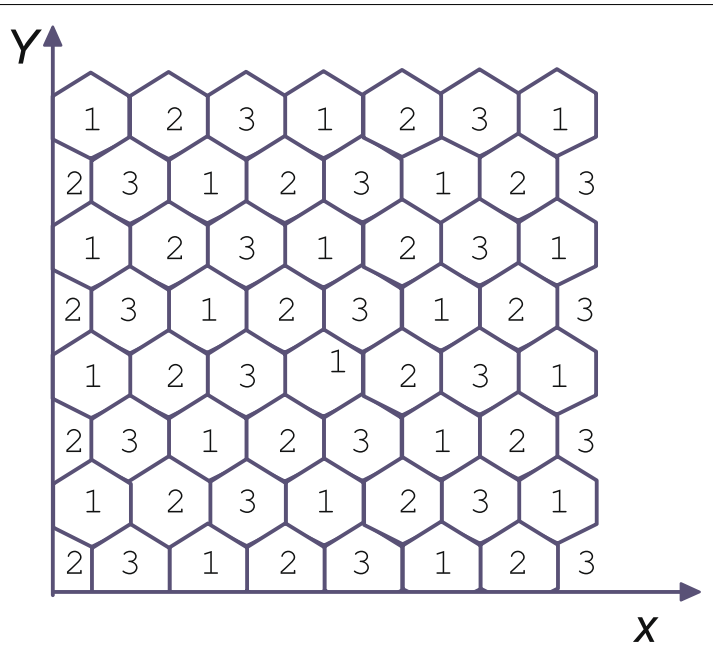

Fig. 1 Network area partition with three different colors 
time slot $t$ by choosing one from each hexagon in the same group. The set of links scheduled simultaneously in time slot $t$ is denoted by $E_{t}$. Recall that the received power at the receiver is a random variable under the Rayleigh fading model. The link $e_{i} \in E_{t}$ transmits successfully with a probability. Therefore, we examine each link $e \in E_{t}$. If the SINR value of $e$ is smaller than $\beta$, then its communication fails. Afterwards, $e$ is rescheduled in the next time slot. In the next algorithm, we use $\operatorname{SINR}_{E_{t}}(e)$ to denote the SINR value of $e$ in the simultaneous transmission set $E_{t}$ in time slot $t$. This procedure is repeated until all the links belonging to the same group are scheduled. Then we consider other groups until all links in the link length class under consideration are scheduled. The pseudo code of the SLSRF algorithm is presented in Algorithm 1.

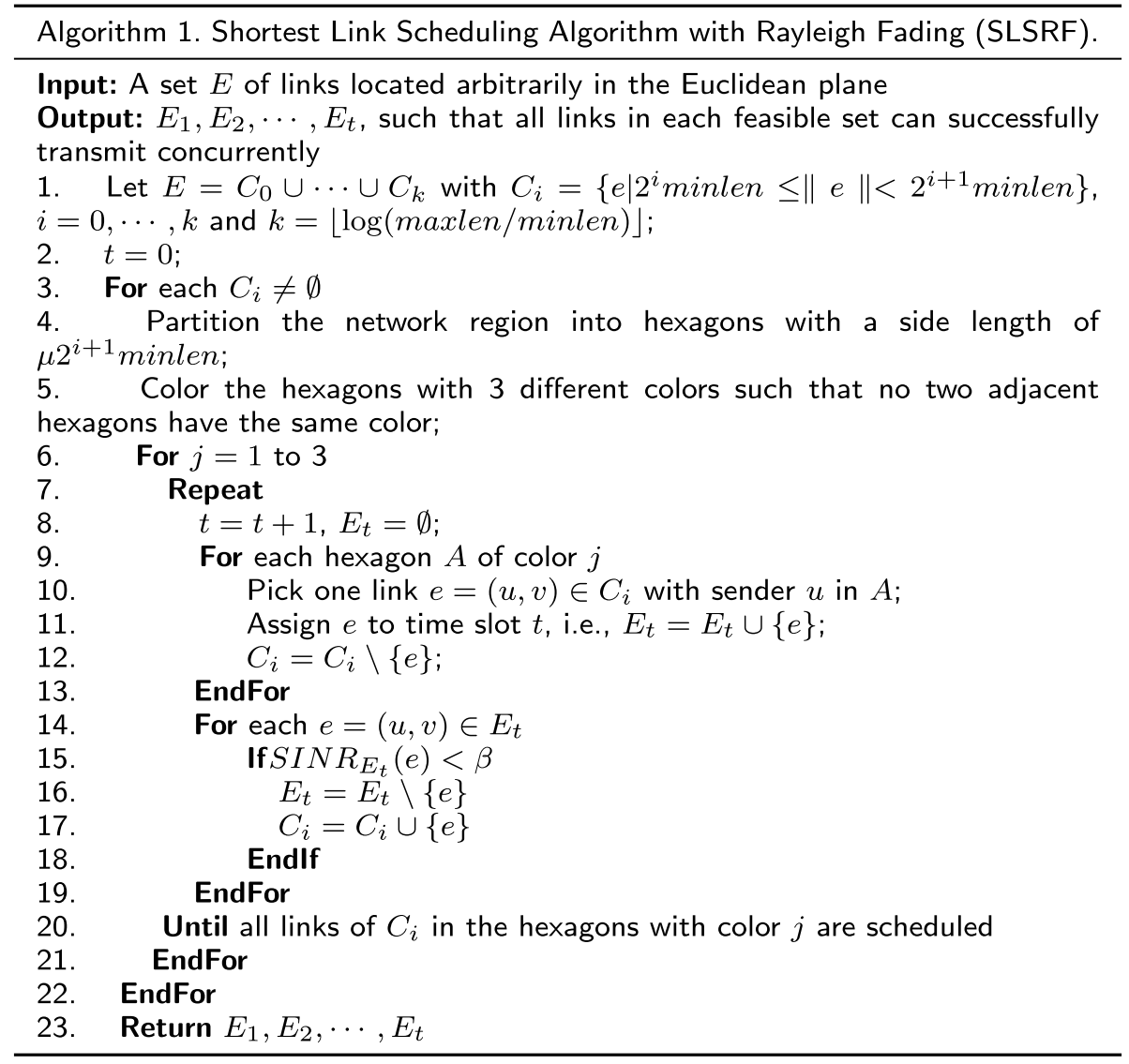

Next, we prove the correctness of algorithm SLSRF.

Lemma 1 [40] If a set $E_{i}$ of links is feasible in the Rayleigh fading channel model with transmitting probability 1 and if the Rayleigh success probability is at least $1 / \sqrt{e}$ for each link, $E_{i}$ is feasible in the SINR model. 
Theorem 2 For each link in $E_{t}, t=1,2, \ldots, T$, the Rayleigh success probability is at least $1 / \sqrt{e}$ with high probability.

Proof Let $e_{i}=\left(u_{i}, v_{i}\right) \in E_{t}$. Then the Rayleigh success probability of $e_{i}$ is

$$
\mathrm{P}=\exp \left(-\beta N Q_{u_{i}}^{-1}\left\|u_{i} v_{i}\right\|^{\alpha}\right) \prod_{e_{j} \in E_{t} \wedge j \neq i}\left(1+\beta\left\|u_{i} v_{i}\right\|^{\alpha}\left\|u_{j} v_{i}\right\|^{-\alpha}\right)^{-1}
$$

Obviously, $\mathrm{P}$ is proportional to $\left\|u_{j} v_{i}\right\|$ and is inversely proportional to $\left\|u_{i} v_{i}\right\|$. That is, if the interference is fixed, the larger the length of $e_{i}$ is, the smaller the Rayleigh success probability is. On the other hand, if $e_{i}$ is fixed, the smaller the distance of $\left\|u_{j} v_{i}\right\|$ is, the smaller the Rayleigh success probability is. This observation means that if $e_{j}$ is near $e_{i}$, then the interference of $e_{j}$ on $e_{i}$ is large and the Rayleigh success probability of $e_{i}$ is small. We conclude that if $\left\|u_{j} v_{i}\right\|$ takes the minimum value and $\left\|u_{i} v_{i}\right\|$ takes the maximum value then $\mathrm{P}$ takes the minimum value. Next, we derive the lower bound of $\mathrm{P}$.

Assume that $u_{i}$ resides in the hexagon labeled A (see Fig. 2), the color of which is 1 . According to the SLSRF algorithm, the senders of the links in $E_{t}$ lie in the hexagons with the same color 1 , and these hexagons form hexagon-shaped belts, as shown in Fig. 2. The first belt, i.e., the inner belt, contains 6 hexagons (black hexagons in Fig. 2, and the distance from the links in these 6 hexagons to $v_{i}$ is at least $(\mu-1) 2^{i+1}$ minlen. More generally, the $h$ th belt contains $6 h$ hexagons. When $h$ is even, the distance from links in the $6 h$ hexagons to $v_{i}$ is at least $\left(\frac{(3 h-2) \sqrt{3} \mu}{2}-1\right) 2^{i+1}$ minlen. When $h$ is odd, the distance from links in the $6 h$ hexagons to $v_{i}$ is at least $\left(\frac{\sqrt{27 h^{2}-36 h+13} \mu}{2}-1\right) 2^{i+1}$ minlen. Since $(3 h-2) \sqrt{3} \mu=\sqrt{27 h^{2}-36 h+12}<\sqrt{27 h^{2}-36 h+13}$, we conclude that the distance from the links in the $h$ th belt hexagons to $v_{i}$ is at least $\left(\frac{(3 h-2) \sqrt{3} \mu}{2}-1\right) 2^{i+1}$ minlen

Therefore,

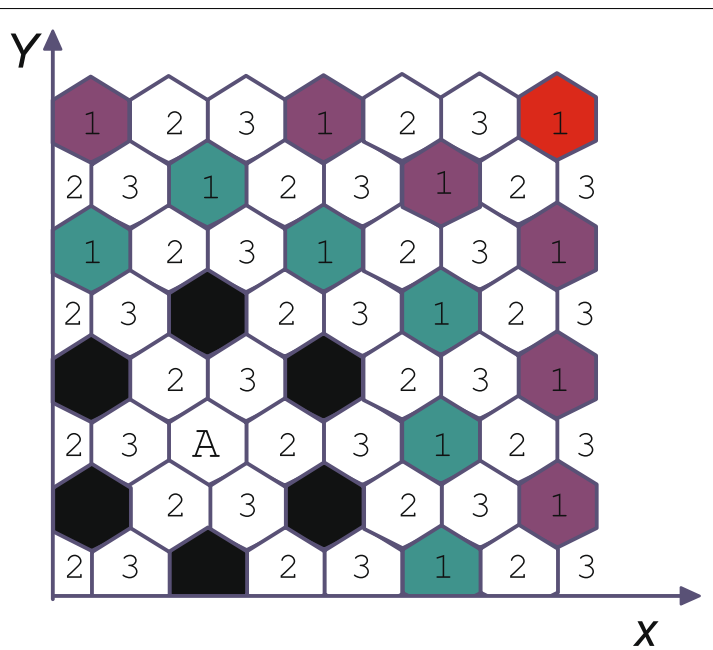

Fig. 2 Select one link from the colored hexagons (with label 1) 


$$
\begin{aligned}
\mathrm{P}= & \exp \left(-\beta N Q_{u_{i}}^{-1}\left\|u_{i} v_{i}\right\|^{\alpha}\right) \prod_{e_{j} \in E_{t} \wedge j \neq i}\left(1+\beta\left\|u_{i} v_{i}\right\|^{\alpha}\left\|u_{j} v_{i}\right\|^{-\alpha}\right)^{-1} \\
\geq & \exp \left(-\beta N Q_{u_{i}}^{-1}\left(2^{i+1} \text { minlen }\right)^{\alpha}\right)\left(1+\beta(\mu-1)^{-\alpha}\right)^{-6} \\
& \prod_{h=2}^{\infty}\left(1+\beta\left(\frac{(3 h-2) \sqrt{3} \mu}{2}-1\right)^{-\alpha}\right)^{-6 h} \\
= & \exp \left(-\frac{1}{8}\right) \cdot \frac{4}{5} \cdot \prod_{h=2}^{\infty}\left(1+\beta\left(\frac{(3 h-2) \sqrt{3} \mu}{2}-1\right)^{-\alpha}\right)^{-6 h} .
\end{aligned}
$$

Let $X=\prod_{h=2}^{\infty}\left(1+\beta\left(\frac{(3 h-2) \sqrt{3} \mu}{2}-1\right)^{-\alpha}\right)^{-6 h}$. Obviously, $X$ is determined by $\alpha, \beta$ and $h$ all together. When $h$ and $\beta$ are fixed, $X$ decreases with decreasing $\alpha$. Similarly, when $h$ and $\alpha$ are fixed, $X$ decreases with increasing $\beta$. Since $2<\alpha<6$, and generally $\beta<40$. Next, we set $\alpha=2$ and $\beta=50$. Then, when $h<48, \mathrm{P}>1 / \sqrt{e}$. That is, when $h<48$, the SLSRF ensures a Rayleigh success probability of each link of no less than $1 / \sqrt{e}$. It seems that the SLSRF is not precise. However, in a real network environment, $\alpha>2$ and $\beta<25$. For instance, if $\alpha=3$ and $\beta=10$, which are typical settings, then $\mathrm{P}$ is always larger than $1 / \sqrt{e}$.

Let $Y=\left(1+\beta\left(\frac{(3 h-2) \sqrt{3} \mu}{2}-1\right)^{-\alpha}\right)^{-6 h}$. Figures 3 and 4 show the variation tendency of $Y$, which is called the interference factor, with the variation of $\alpha, \beta$ and $h$. From Fig. 3, we can derive that when $h>12$, the interference factors reaches up to 1 . From Fig. 4, we can see that different $\beta$ values have almost the same influence on the Rayleigh success probability of $e_{i}$. When $h \geq 12$, the interference factors reach up to 1 . Therefore, we can conclude that when $h \geq 12$, the interference factors can be neglected and they do not impact the Rayleigh success probability of $e_{i}$. The Rayleigh success probability of $e_{i}$ is $\mathrm{P}>0.8825 \times 0.8 \times 0.99^{\mathrm{h}}$. When $h \leq 12, \mathrm{P}>1 / \sqrt{\mathrm{e}}$. However, when $h>12$, the influence factors of the $h$ th links can be neglected. Therefore, for each link in $E_{t}$, $t=1,2, \ldots, T$, the Rayleigh success probability is at least $1 / \sqrt{e}$ with high probability.

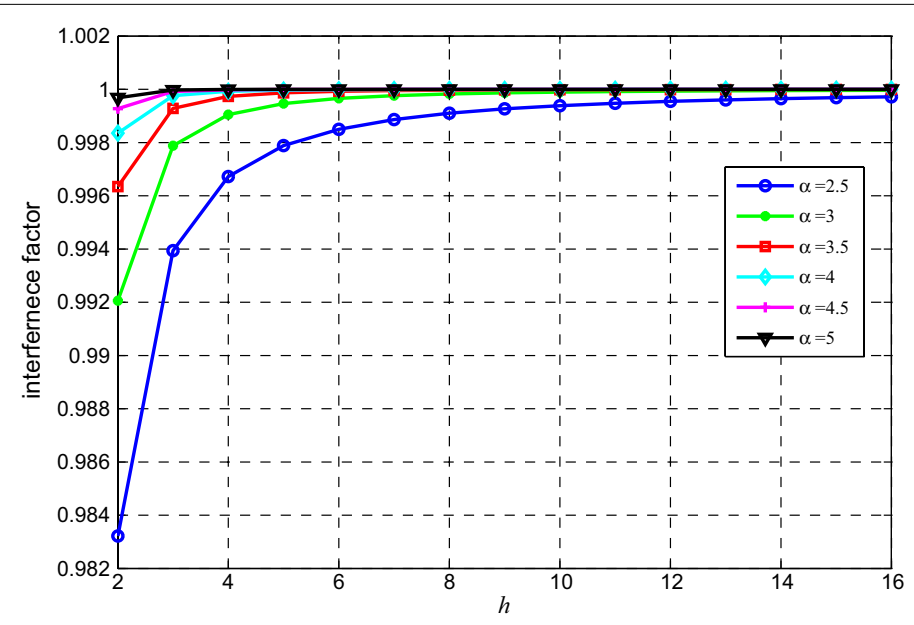

Fig. $3 Y$ increases sharply to 1 with different $\alpha$ s, $\beta=10$ 


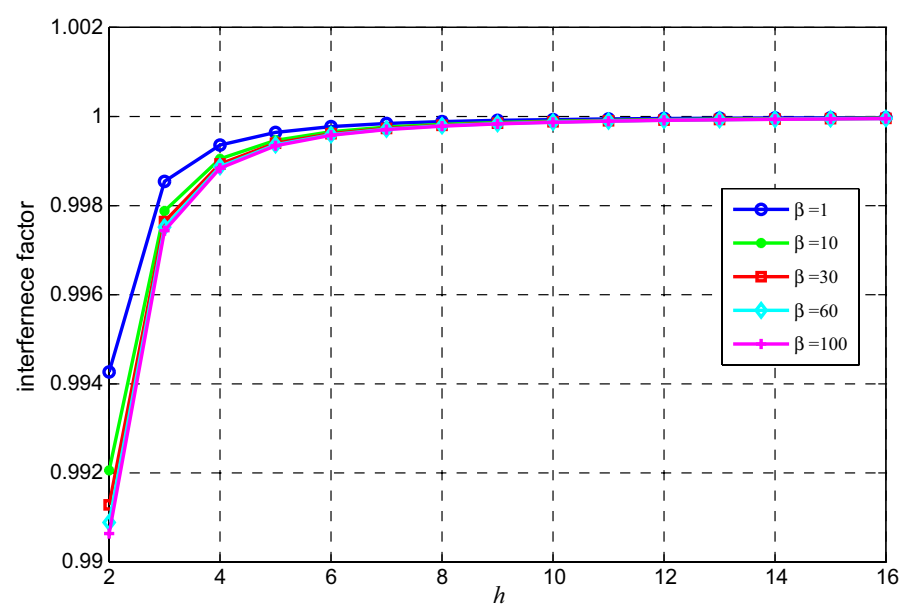

Fig. $4 Y$ increases sharply to 1 with different $\beta$ s, $\alpha=3$

\subsection{Discussion}

In the Rayleigh fading channel model, the successful transmission of a link is a random variable. Although the Rayleigh success probability of $e_{i}$ is smaller than $1 / \sqrt{e}$, the transmission of $e_{i}$ may be successful. Therefore, $E_{t}$ is a correct feasible set even though the Rayleigh success probabilities of some links are lightly smaller than $1 / \sqrt{e}$. The Rayleigh success probabilities of $e_{i}$ is impacted by many factors. For instance, in an application environment, the size of the network is finite, that is, $h$ is generally small. Moreover, for the proof of Theorem 2, we assume that one link is selected from hexagons with the same color as that of the one that $e_{i}$ resides in. In fact, there may be no links to be selected. These factors make the Rayleigh success probability of $e_{i}$ larger than that analyzed in Theorem 2. On the other hand, if we improve the transmitting power, such as $Q_{i}=10 \beta N\left(2^{i+1} \text { minlen }\right)^{\alpha}$, the first factor of the Rayleigh success probability increases and the Rayleigh success probability of $e_{i}$ increases. Therefore, we can improve the transmitting power if necessary.

\section{Simulations}

Next, we show the correctness and effectiveness of the proposed algorithm by simulations. Comparing the SLSRF with the SLSPC and SLSUM [22], we conclude that the SLSRF is indeed efficient.

\subsection{Simulation settings}

Assume that all nodes are deployed in a large network region with an area of $2000 \mathrm{~m} \times 2000 \mathrm{~m}$ randomly and that the distance between each pair of nodes is no less than 1 . We further assume that the link lengths are arbitrarily distributed; thus, we randomly select two points, say $u_{i}$ and $v_{i}$, forming a link $e_{i}=\left(u_{i}, v_{i}\right)$ in the network region that satisfies the condition minlen $\leq\left\|u_{i} v_{i}\right\| \leq$ maxlen. In the following simulations, we let minlen $=1$ and maxlen $=30$. Assume that each link has a distinct sender and a distinct receiver. The ambient noise $N$ is fixed at $-70 \mathrm{db}$. We set $\alpha=3$ and $\beta=10 \mathrm{db}$ unless the impact of a parameter is under consideration, in which case we adjust the 
Table 1 Parameter settings

\begin{tabular}{llll}
\hline Parameter & Value & Parameter & Value \\
\hline Network area & $2000 \mathrm{~m} \times 2000 \mathrm{~m}$ & $\beta$ & $\geq 1$ \\
\#links & $200-1000$ & $N$ & $-70 \mathrm{db}$ \\
minlen & 1 & $\varepsilon_{1}[33]$ & 0.4 \\
maxlen & 30 & $\varepsilon_{2}[22]$ & 4 \\
$\alpha$ & $2-6$ & $\varphi[22]$ & 2.5 \\
\hline
\end{tabular}

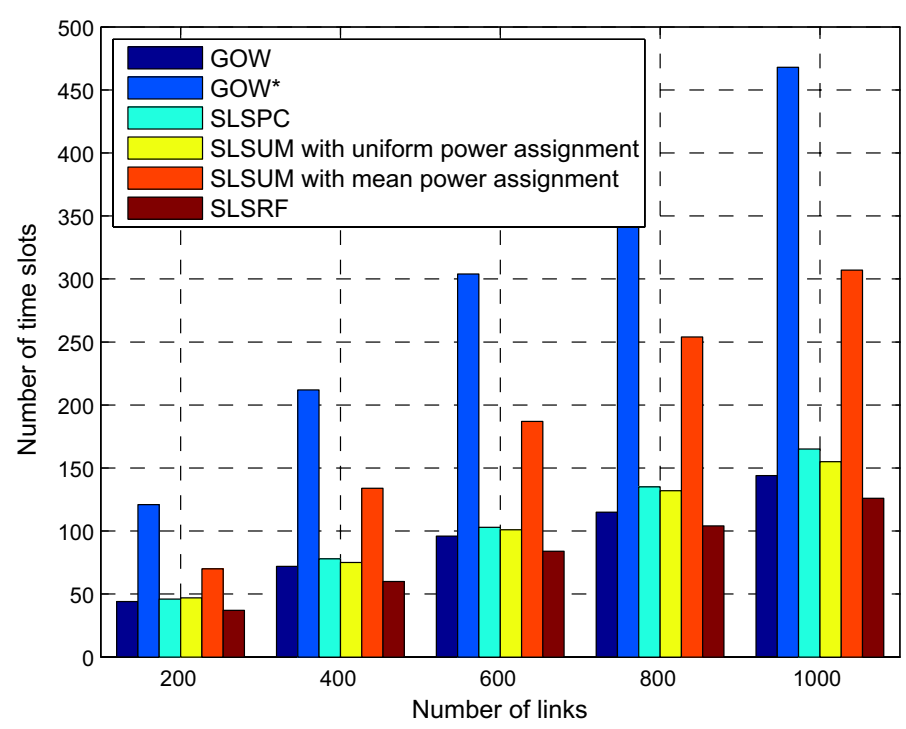

Fig. 5 The SLSRF has the best performance

corresponding parameter within a reasonable value range. We compare the SLSRF with four algorithms: GOW [32], GOW* [33], SLSPC and SLSUM [22]. Some parameters influence the scheduling results of the GOW*, SLSPC and SLSUM, and we set $\varepsilon_{1}=0.4$ (in [33] $\varepsilon_{1} \geq 1 / 7$ ) for the GOW*, $\varepsilon_{2}=4$ (in [22] $\varepsilon_{2}>0$ ) for the SLSPC and $\varphi=2.5$ (in [22] $\varphi>0$ ) for the SLSUM. We choose $\varepsilon_{1}=0.4, \varepsilon_{2}=4$ and $\varphi=2.5$ because these settings yield the best performance in terms of latency based on our simulations on the GOW*, SLSPC and SLSUM. The important parameters are listed in Table 1.

\subsection{Simulation results}

Figure 5 demonstrates that in the case of the same parameter settings, the SLSRF has the shortest latency among all the algorithms. Although GOW does not consider ambient noise, the SLSRF has better performance than that of the GOW. The ideas behind the SLSRF, SLSUM and SLSPC are similar, but the SLSRF has the best performance, since the side length of hexagons of the SLSRF is the smallest. Then, the SLSRF partitions the network area into hexagons with the largest number and selects a set of links with the largest number. Therefore, the SLSRF under the Rayleigh fading channel model has better performance than that of the SLSPC and SLSUM under the SINR model. 
Next, we investigate the influence of the parameters on the SLSRF. The main influence factors are $\alpha$ and $\beta$. We consider a link, say $e=(u, v)$. With increasing $\alpha$, the interference at the receiver $v$ decreases. However, the intended mean power does not vary. Therefore, intuitively, the links causing mutual interference are allowed to be close to each other. Indeed, with increasing $\alpha, \mu$ decreases, and the distances between links in the same time slot become small, the number of hexagons becomes large, and the number of links in the same time slot becomes large. Therefore, the latency of the set of links decreases, which corresponds to Fig. 6.

Similarly, with the increase in $\beta$, the interference at the receiver must decease; thus, the distance between links transmitting simultaneously should increase. Indeed, with increasing $\beta, \mu$ increases, and the side length of hexagons also increases. Therefore, the latency of the set of links increases, which corresponds to Fig. 7. The number of links is 400 in Figs. 6 and 7.

Finally, we examine the Rayleigh success probability of each link in a time slot. According to Theorem 2 , the Rayleigh success probability of each link is at least $1 / \sqrt{e}$. However, if a link transmits a signal successfully, then its SINR value should be at least $\beta$. First, we select a link set, denoted by $E_{R}$, in which the Rayleigh success probability of each link is at least $1 / \sqrt{e}$. Then, we calculate the SINR value of each link. The link whose SINR value is larger than $\beta$ is selected to join link set $E_{S}$. The ratio of $E_{S}$ to $E_{R}$ is defined as the success ratio. We set $\alpha=3, \beta=10 \mathrm{db}$ and \#links $=800$. The program runs 30 times independently. The total number of time slots is 3150 . The number of time slots in which the success ratio is smaller than $1 / \sqrt{e}$ is 24 , which accounts for $0.76 \%$ the total time slots. The number of time slots in which the success ratio is between $1 / \sqrt{e}$ and $100 \%$ is 23 . Therefore, the proportion of time slots with a $100 \%$ success ration is $98.5 \%$. Table 2 illustrates the above results.

\section{Distributed implementation}

For a large wireless network, a distributed link scheduling protocol is more practical than a centralized one because a centralized protocol requires more information about the structure and topology of the network. For example, the SLSRF needs a central

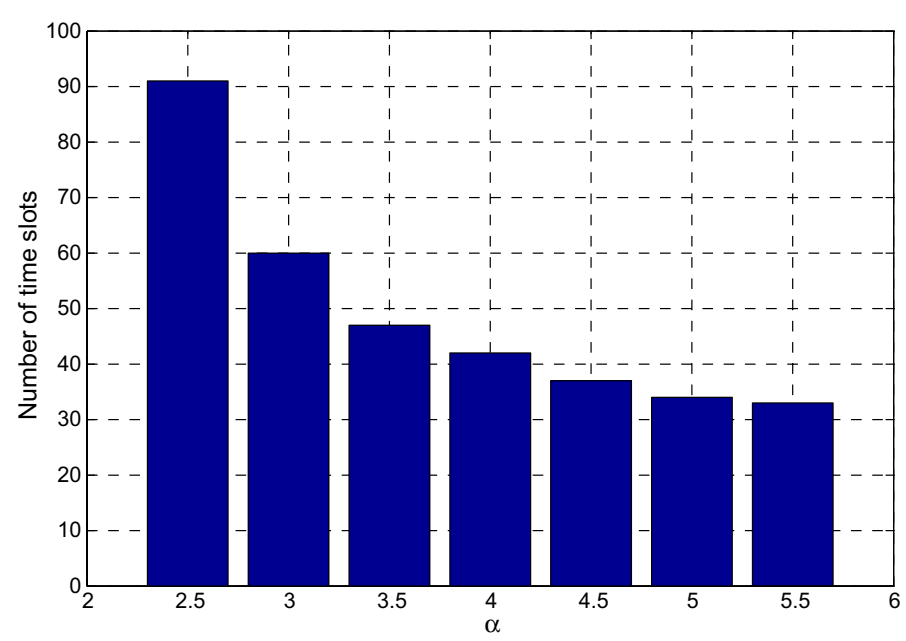

Fig. 6 Influence of $\alpha$ on the SLSRF 


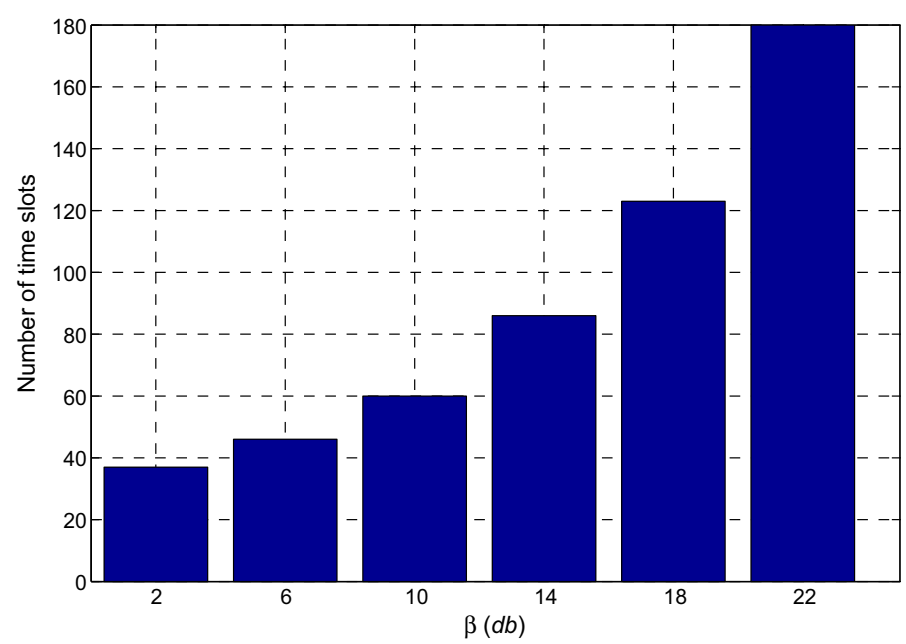

Fig. 7 Influence of $\beta$ on the SLSRF

Table 2 Success ratio

\begin{tabular}{lcl}
\hline Success ratio (\%) & Number of slots & Proportion (\%) \\
\hline 100 & 3103 & 98.5 \\
$60-100$ & 23 & 0.74 \\
$<60$ & 24 & 0.76 \\
\hline
\end{tabular}

processing unit to collect the information of links, such as the position, number of hexagons, etc. Additionally, the central processing unit must schedule one link from a hexagon and then inform the result to all other links. Therefore, we consider a distributed implementation of the SLSRF, named DI-SLSRF, since all hexagons with the same color are separated and the links in different hexagons can be scheduled in a distributed way.

\subsection{Network model and assumptions}

Recall that we use a graph $G=(V, E)$ to model a wireless network, with $V$ being the transceivers and $E$ being the set of links. Assume that all nodes are deployed in a 2-dimensional plane area that has a central position. The assumption is reasonable and matches the case of practical wireless networks, such as cell-networks with base stations or wireless networks with access points (APs). Assume each node knows the central position and its own position, which is done by space positioning techniques. Another assumption is that a sender knows the target position. In this case, a sender is aware of the distance of its communication. We normalize the minimum distance 1 , that is, minlen $=1$. Again, we use maxlen to denote the maximum communication distance. In this section, we assume a synchronization system in which the time is divided into slots.

\subsection{Outline of the DI-SLSRF algorithm}

We define a link class $C_{i}, i=0,1,2, \ldots,\lfloor\log$ maxlen $\rfloor$, in which the length of each link $e$ satisfies $2^{i} \leq\|e\|<2^{i+1}$. The senders are aware of which class they belong to, since they know the distance of their communication. 
First, the network area is partitioned into hexagons with a side length of $2^{i+1} \mu$ for the $i$ th group, $\mu$ is defined as Eq. (9). Without loss of generality, we set the central position as the origin of the coordinate system and the center point of a hexagon. We label the central hexagon 1, the top-left hexagon 2 and the top-right hexagon 3 . All hexagons are labeled 1, 2 or 3 such that no two neighboring hexagons have the same labels; see Fig. 8. As shown in Fig. 1, we use 1, 2 and 3 to denote three different colors. Obviously, the senders are aware of the color of the hexagon in which they reside.

Again, we note that the DI-SLSRF schedules at most one link from a hexagon. That is, at most one sender sends a message in one hexagon. For this reason, all senders residing in the same hexagon compete for the channel to send messages. Therefore, we design a subroutine, named the leader election (LE) algorithm, see Algorithm 2, to select at most one sender to send a message. The LE comprises $\theta \log n$ rounds, and each round contains four slots, where $n$ denotes the number of senders. Assume a message can be received by the receiver in one slot time.

A sender has 4 different states: competitor, candidate, loser and leader. In the competitor state, a sender sends a probe message with probability $p=1 / n$ for the purpose of competing for the channel in the first slot and receives the message in the second slot. If a sender sends a probe message and does not receive any probe messages, it changes its state to the candidate, which means that it will occupy the channel. On the other hand, if a sender sending a probe message receives at least a probe message, which means that two or more senders compete for the channel, then it keeps its state and senses the channel in the next slot. In the candidate state, a sender informs other senders that it will occupy the channel by sending a busy message in the third slot and listening to the fourth slot. If a sender in the candidate state receives a busy message, which means that another sender occupies the channel, it changes its state to the loser and no longer competes for the channel. If it does not receive any busy messages, it changes its state to the leader. In the leader state, a sender occupies the channel and sends its message to the receiver. That is, the link with the sender in the leader state is scheduled. After being scheduled, the node quits the LE algorithm. At the beginning

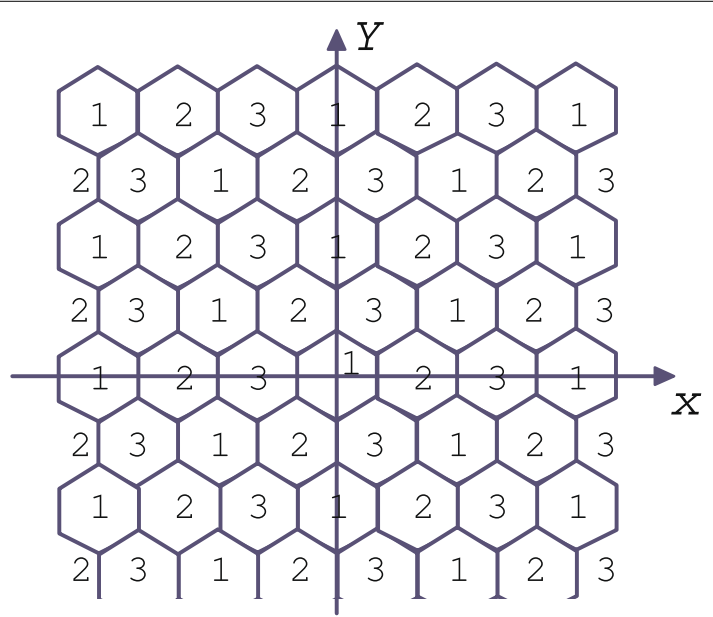

Fig. 8 The central position overlaps the origin of the coordinate system 
of the algorithm, all senders are initialized with the competitor state. The state diagram is shown in Fig. 9, and the pseudo code is listed in Algorithm 2.

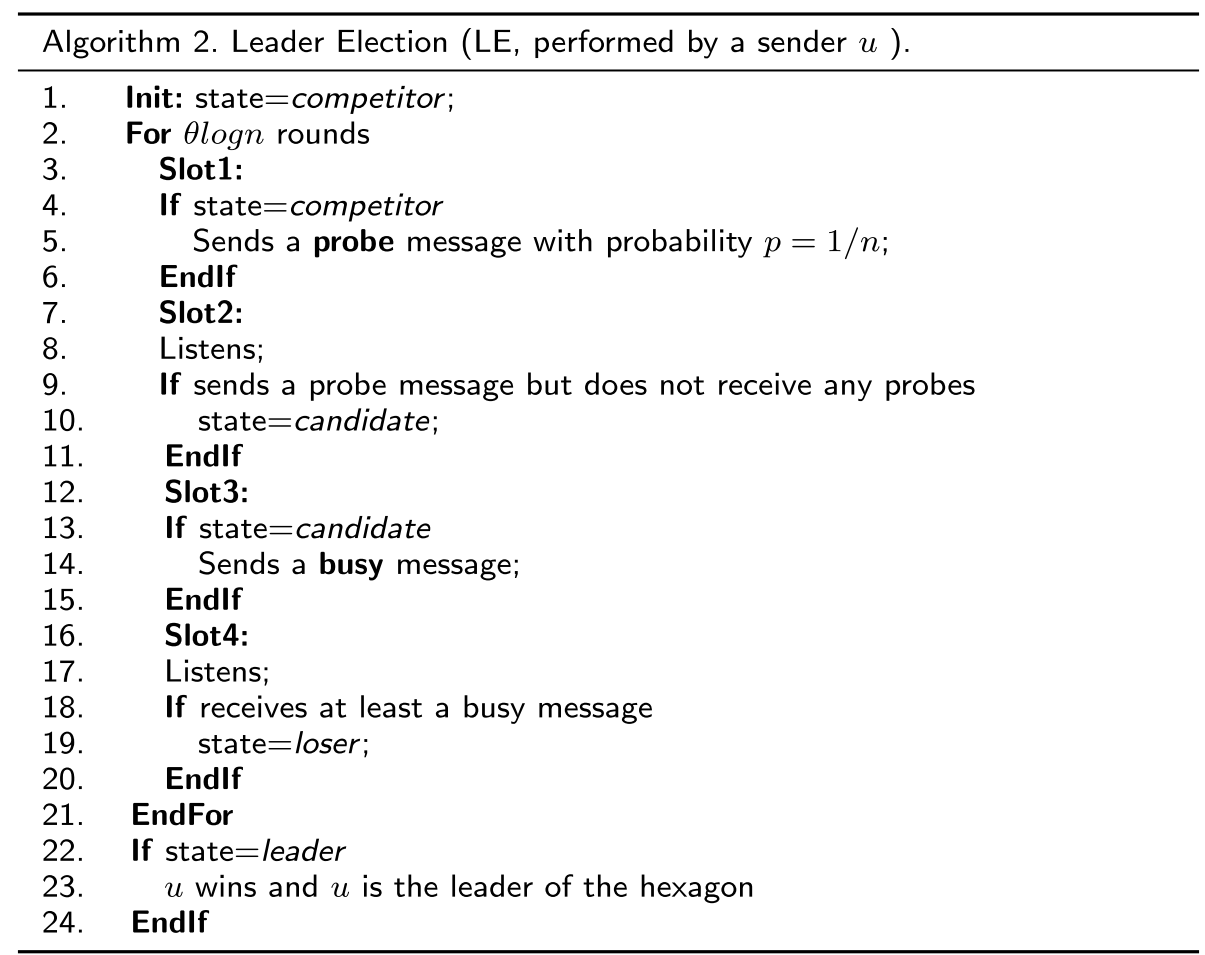

Slot1: send probe message with a probability Slot2: listen

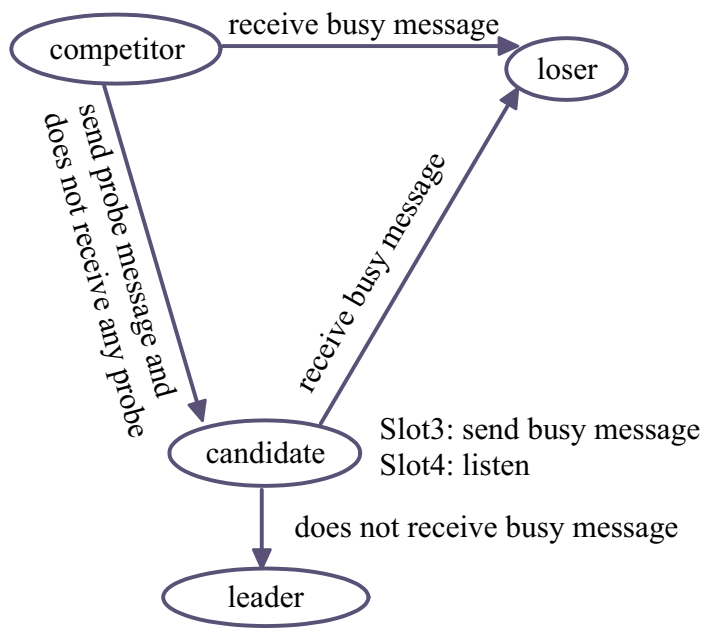

Fig. 9 State diagram 
Next, we provide the outline of the DI-SLSRF. We define a notation Super-Big-Round, which comprises 3 Big-Rounds. A Big-Round comprise $4 \theta \log n+1$ slots. All senders residing in the hexagons of the same color, say 1, execute the subroutine LE in a distributed way, using $4 \theta \log n$ slots. After executing the subroutine LE, at most one sender in each hexagon with color 1 is selected as the leader. All leaders send messages to the receivers in the next slot. Then, senders residing in the same color hexagons, say 2 and 3, execute the LE algorithm, and the selected leaders send messages to their receivers. We call this process a Super-Big-Round, which corresponds to a class. If there are unscheduled links, the DI-SLSRF is carried out continuously. Initially, let $i=0$. The pseudo code of the DI-SLSRF is presented in Algorithm 3 .

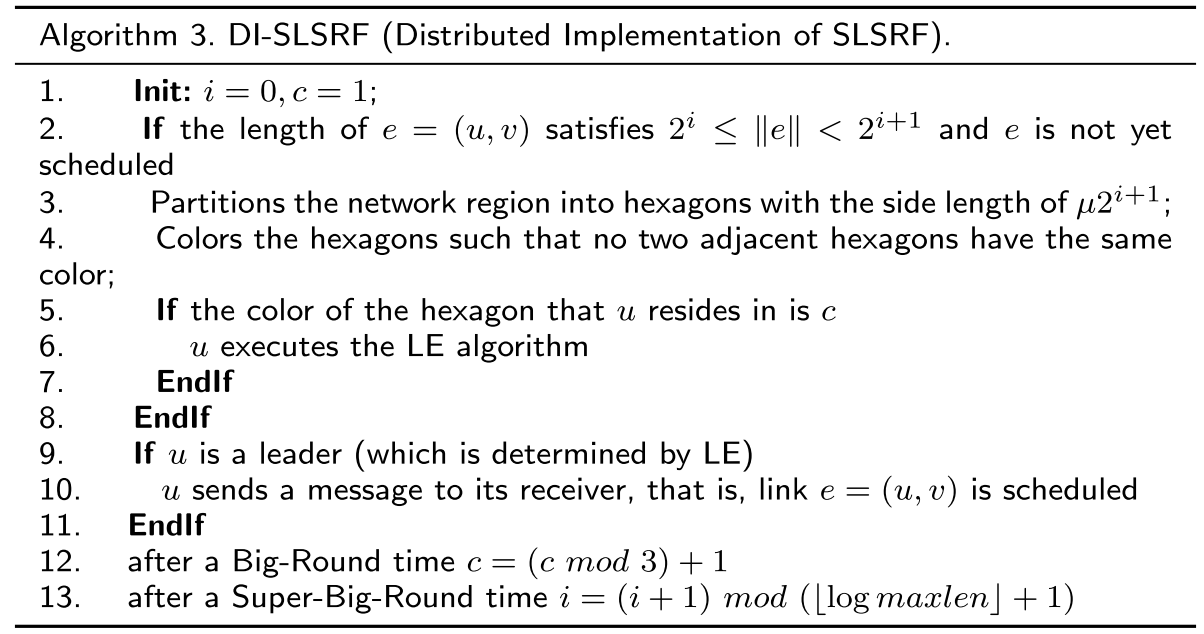

\subsection{Analysis}

Next, we prove the correctness of the DI-SLSRF.

Lemma 2 For each hexagon, at most one sender with the leader state is returned by the $L E$, that is, no two senders transmit data to their receivers concurrently.

Proof If $u$ sends a probe message in the first slot and does not receive any probe messages in the second slot, then no other sender competes with $u$ for the channel; hence, $u$ will occupy the channel in the following slot. On the other hand, once $u$ occupies the channel, $u$ sends a busy message to other senders, and the senders sensing the channel busy will change their states to the loser and no longer compete for the channel. In summary, only one sender occupies the channel at the end of the LE algorithm.

Lemma 3 The LE selects one sender with the leader state with a high probability.

Proof Assume that the LE does not select one sender in a round $k$; there are two events leading to this result. One is the event where two or more senders send probe messages 
to compete for the channel. The other is where no sender sends a probe message. In summary, the probability of the event in which the LE does not select one sender in a round is $1-p(1-p)^{\zeta}$, where $\zeta$ is the number of senders executing the LE simultaneously.

Next, we give the following fact.

Fact: Given a set of probabilities $p_{1}, p_{2}, \ldots, p_{n}$, with $\forall i, p_{i} \in[0,1 / 2]$, the following inequalities hold:

$$
(1 / 4)^{\sum_{k=1}^{n} p_{k}} \leq \prod_{k=1}^{n}\left(1-p_{k}\right) \leq(1 / e)^{\sum_{k=1}^{n} p_{k}}
$$

After $\theta \log n$ rounds, the probability of the LE not selecting one sender is at most

$$
p_{\text {no }} \leq(1-p / 4)^{\theta \log n} \leq e^{-\frac{p \theta}{4} \log n} \in n^{-5} .
$$

The last equation holds only if $\theta$ is sufficiently large. That is, after $\theta \log n$ rounds, the LE algorithm selects one sender with the leader state with the probability $1-n^{-5}$, which is a high probability.

Theorem 3 Without loss of generality, assume that the link $e=(u, v)$ belongs to the $i$-th link class and resides in a hexagon with color $c=1$. If $u$ is the only leader returned by the $L E$, then the Rayleigh success probability is at least $1 / \sqrt{e}$ with high probability.

Proof $u$ is the only link returned by the LE in the hexagon with color 1 . The next proof is the same as that of Theorem 2. Note that, the correctness of Theorem 2 is under the assumption that a link must be scheduled in a hexagon. In the DI-SLSRF, there may be a link scheduled in a hexagon. Therefore, Theorem 2 guarantees the correctness of this theorem.

\section{Conclusions}

In this paper, we study the shortest link scheduling problem under the Rayleigh fading model and propose the SLSRF and DI-SLSRF algorithms. The SLSRF partitions the network region into hexagons and colors them with three different colors such that no two adjacent hexagons have the same color. Then, the SLSRF selects one link from a hexagon with a certain color. A theoretical analysis proves that the links selected from the hexagons with the same color are scheduled simultaneously without interference. The DI-SLSRF is a distributed version of the SLSRF, that is more suitable for large-scale networks.

A drawback of offline link scheduling is that it neglects the dynamic nature of request scheduling in wireless networks. Therefore, online link scheduling in wireless networks with the Rayleigh fading model is an open problem. 


\section{Abbreviations}

5G: The fifth-generation; URLLC: Ultrareliable low-latency communications; SINR: Signal-to-interference plus noise ratio; MWISL: Maximum-weighted independent set of links; SLSRF: The shortest link scheduling with Rayleigh fading model; DI-SLSRF: Distributed implementation of the SLSRF; LE: Leader election.

\section{Acknowledgements}

This work is supported by the National Natural Science Foundation of China Grants 61672321, 61832012, 61771289 and 61373027, Shandong Province Fundamental Research (Grant No. ZR201906140028) and Shandong Province Key Research and Development Plan (Grant No. 2019GGX101050).

\section{Authors' Contributions}

$\mathrm{BH}, \mathrm{FL}$ and $\mathrm{CM}$ are the principal contributors in terms of simulation modelling and the generation/interpretation of numerical results. In a supervising role, JY and GL formulated the research problem and contributed to the simulation modelling and the discussion of results. All authors read and approved the final manuscript.

\section{Funding}

The work is supported by the National Natural Science Foundation of China, Shandong Province Fundamental Research and Shandong Province Key Research and Development Plan.

\section{Availability of data and materials}

Data sharing not applicable to this article as no data sets were generated or analyzed during the current study.

\section{Declarations}

\section{Competing interests}

The authors declare that they have no competing interests.

\section{Author details}

${ }^{1}$ School of Computer Science, Qufu Normal University, Rizhao, China. ${ }^{2}$ School of Computer Science and Technology, Qilu University of Technology (Shandong Academy of Sciences), Jinan, China. ${ }^{3}$ Shandong Computer Science Center (National Supercomputer Center in Jinan), Jinan, China. ${ }^{4}$ Shandong Provincial Key Laboratory of Computer Networks, Jinan, China.

Received: 29 March 2021 Accepted: 1 June 2021

Published online: 10 June 2021

\section{References}

1. J. Mabrouki, M. Azrour, D. Dhiba, Y. Farhaoui, S.E. Hajjaji, IOT-based data logger for weather monitoring using Arduino-based wireless sensor networks with remote graphical application and alerts. Big Data Min. Anal. 4(1), 25-32 (2021). https://doi.org/10.26599/BDMA.2020.9020018

2. J. Mabrouki, M. Azrour, G. Fattah, D. Dhiba, S.E. Hajjaji, Intelligent monitoring system for biogas detection based on the internet of things: Mohammedia, morocco city landfill case. Big Data Min. Anal. 4(1), 10-17 (2021). https://doi. org/10.26599/BDMA.2020.9020017

3. Z. Zhang, X. Cong, W. Feng, H. Zhang, G. Fu, J. Chen, Waeas: An optimization scheme of EAS scheduler for wearable applications. Tsinghua Sci. Technol. 26(1), 72-84 (2021). https://doi.org/10.26599/TST.2019.9010040

4. Y. Fu, Y. Hou, Z. Wang, X. Wu, K. Gao, L. Wang, Distributed scheduling problems in intelligent manufacturing systems. Tsinghua Sci. Technol. 26(5), 625-645 (2021). https://doi.org/10.26599/TST.2021.9010009

5. Y. Xiao, Y. Jia, C. Liu, X. Cheng, J. Yu, W. LV, Edge computing security: state of the art and challenges. Proc. IEEE 107(8), 1608-1631 (2019). https://doi.org/10.1109/JPROC.2019.2918437

6. L. Qi, X. Wang, X. Xu, W. Dou, S. Li, Privacy-aware cross-platform service recommendation based on enhanced locality-sensitive hashing. IEEE Trans. Netw. Sci. Eng. (2020)

7. A. Zhao, J. Li, M. Ahmed, Spidernet: A spiderweb graph neural network for multi-view gait recognition. Knowl. Based Syst. 206, 106273 (2020). https://doi.org/10.1016/j.knosys.2020.106273

8. L. Qi, C. Hu, X. Zhang, M.R. Khosravi, S. Sharma, S. Pang, T. Wang, Privacy-aware data fusion and prediction with spatial-temporal context for smart city industrial environment. IEEE Trans. Ind. Inform. 17(6), 4159-4167 (2021). https://doi.org/10.1109/TII.2020.3012157

9. H. Kou, H. Liu, Y. Duan, W. Gong, Y. Xu, X. Xu, L. Qi, Building trust/distrust relationships on signed social service network through privacy-aware link prediction process. Appl. Soft Comput. 100, 106942 (2021). https://doi.org/10. 1016/j.asoc.2020.106942

10. F. Wang, H. Zhu, G. Srivastava, S. Li, M. Khosravi, L. Qi, Robust collaborative filtering recommendation with user-itemtrust records. IEEE Trans. Comput. Soc. Syst. 1-11 (2021). https://doi.org/10.1109/TCSS.2021.3064213

11. Y. Liu, F. Wang, Y. Yang, X. Zhang, H. Wang, H. Dai, L. Qi, An attention-based category-aware GRU model for next poi recommendation. Int. J. Intell. Syst. 1-11 (2021). https://doi.org/10.1002/int.22412

12. R. Bi, Q. Liu, J. Ren, G. Tan, Utility aware offloading for mobile-edge computing. Tsinghua Sci. Technol. 26(2), 239-250 (2021). https://doi.org/10.26599/TST.2019.9010062

13. M. Azrour, J. Mabrouki, A. Guezzaz, Y. Farhaoui, New enhanced authentication protocol for internet of things. Big Data Min. Anal. 4(1), 1-9 (2021). https://doi.org/10.26599/BDMA.2020.9020010

14. G. Pocovi, K.I. Pedersen, P. Mogensen, Joint link adaptation and scheduling for $5 \mathrm{G}$ ultra-reliable low-latency communications. IEEE Access 6, 28912-28922 (2018). https://doi.org/10.1109/ACCESS.2018.2838585

15. M. Saad, S. Abdallah, On millimeter wave 5 G backhaul link scheduling. IEEE Access 7, 76448-76457 (2019). https:// doi.org/10.1109/ACCESS.2019.2922146 
16. N. Chen, Z. Wang, R. He, J. Jiang, F. Cheng, C. Han, Efficient scheduling mapping algorithm for row parallel coarsegrained reconfigurable architecture. Tsinghua Sci. Technol. 26(5), 724-735 (2021). https://doi.org/10.26599/TST.2020. 9010035

17. T. Moscibroda, R. Wattenhofer, The complexity of connectivity in wireless networks. in INFOCOM 2006. 25th IEEE International Conference on Computer Communications, Joint Conference of the IEEE Computer and Communications Societies, 23-29 April 2006 (Barcelona, Catalunya, Spain, 2006), pp. 76448-76457. https://doi.org/10.1109/INFOCOM. 2006.23

18. O. Goussevskaia, M.M. Halldórsson, R. Wattenhofer, Algorithms for wireless capacity. IEEE/ACM Trans. Netw. 22(3), 745-755 (2014). https://doi.org/10.1109/TNET.2013.2258036

19. B. Huang, J. Yu, D. Yu, C. Ma, SINR based maximum link scheduling with uniform power in wireless sensor networks. TIIS 8(11), 4050-4067 (2014). https://doi.org/10.3837/tiis.2014.11.022

20. H. Deng, J. Yu, D. Yu, G. Li, B. Huang, Heuristic algorithms for one-slot link scheduling in wireless sensor networks under SINR. IJDSN 11, 806520-18065209 (2015). https://doi.org/10.1155/2015/806520

21. Z. Beheshtifard, M.R. Meybodi, Maximal throughput scheduling based on the physical interference model using learning automata. Ad Hoc Netw. 45, 65-79 (2016). https://doi.org/10.1016/j.adhoc.2016.02.020

22. J. Yu, B. Huang, X. Cheng, M. Atiquzzaman, Shortest link scheduling algorithms in wireless networks under the SINR model. IEEE Trans. Veh. Technol. 66(3), 2643-2657 (2017). https://doi.org/10.1109/TVT.2016.2580379

23. J. Wu, D. Lin, G. Li, Y. Liu, Y. Yin, Distributed link scheduling algorithm based on successive interference cancellation in MIMO wireless networks. Wirel. Commun. Mob. Comput. 2019, 9083282-1908328212 (2019). https://doi.org/10. $1155 / 2019 / 9083282$

24. Y. Zou, D. Yu, L. Wu, J. Yu, Y. Wu, Q. Hua, F.C.M. Lau, Fast distributed backbone construction despite strong adversarial jamming. in 2019 IEEE Conference on Computer Communications, INFOCOM 2019 (Paris, France, April 29-May 2, 2019), pp. 1027-1035. https://doi.org/10.1109/INFOCOM.2019.8737477

25. X. Xu, Y. Chen, S. He, P.O. Bobbie, OWLS: opportunistic wireless link scheduling with SINR constraints. in Wireless Algorithms, Systems, and Applications: 14th International Conference, WASA 2019, Proceedings (Honolulu, HI, USA, June 24-26, 2019), pp. 419-431. https://doi.org/10.1007/978-3-030-23597-0_34

26. D. Chizhik, J. Ling, P.W. Wolniansky, R.A. Valenzuela, N. Costa, K. Huber, Multiple-input-multiple-output measurements and modeling in Manhattan. IEEE J. Sel. Areas Commun. 21(3), 321-331 (2003). https://doi.org/10.1 109/JSAC. 2003.809457

27. K. Yu, Y. Wang, J. Yu, D. Yu, X. Cheng, Z. Shan, Localized and distributed link scheduling algorithms in IOT under Rayleigh fading. Comput. Netw. 151, 232-244 (2019)

28. C. García-Corrales, U. Fernández-Plazaola, F.J. Cañete, J.F. Paris, F.J. López-Martínez, Unveiling the hyper-Rayleigh regime of the fluctuating two-ray fading model. IEEE Access 7, 75367-75377 (2019). https://doi.org/10.1109/ ACCESS.2019.2921632

29. D. Bera, I. Chakrabarti, S.S. Pathak, Modelling of cooperative spectrum sensing over Rayleigh fading without CSI in cognitive radio networks. Wirel. Pers. Commun. 86(3), 1281-1297 (2016). https://doi.org/10.1007/s11277-015-2988-8

30. C.A. Gutiérrez, J.J. Jaime-Rodriguez, J.M. Luna-Rivera, D.U. Campos-Delgado, J.V. Castillo, Modeling of non-WSSUS double-Rayleigh fading channels for vehicular communications. Wirel. Commun. Mob. Comput. 2017 (2017). https://doi.org/10.1155/2017/6394653

31. J. Yu, K. Yu, D. Yu, W. Lv, X. Cheng, H. Chen, W. Cheng, Efficient link scheduling in wireless networks under Rayleighfading and multiuser interference. IEEE Trans. Wirel. Commun. 19(8), 5621-5634 (2020). https://doi.org/10.1109/ TWC.2020.2994998

32. O. Goussevskaia, Y.A. Oswald, R. Wattenhofer, Complexity in geometric SINR. in Proceedings of the 8th ACM International Symposium on Mobile Ad Hoc Networking and Computing, MobiHoc 2007 (Montreal, Quebec, Canada, September 9-14, 2007), pp. 100-109. https://doi.org/10.1145/1288107.1288122

33. D.M. Blough, G. Resta, P. Santi, Approximation algorithms for wireless link scheduling with SINR-based interference. IEEE/ACM Trans. Netw. 18(6), 1701-1712 (2010). https://doi.org/10.1109/TNET.2010.2047511

34. X. Xu, S. Tang, P. Wan, Maximum weighted independent set of links under physical interference model. in Wireless Algorithms, Systems, and Applications, 5th International Conference, WASA 2010, Proceedings (Beijing, China, August 15-17, 2010). pp. 68-74. https://doi.org/10.1007/978-3-642-14654-1_8

35. Y. Zhou, X. Li, M. Liu, X. Mao, S. Tang, Z. Li, Throughput optimizing localized link scheduling for multishop wireless networks under physical interference model. IEEE Trans. Parallel Distrib. Syst. 25(10), 2708-2720 (2014). https://doi. org/10.1109/TPDS.2013.210

36. C. Wang, J. Yu, D. Yu, B. Huang, S. Yu, An improved approximation algorithm for the shortest link scheduling in wireless networks under SINR and hypergraph models. J. Comb. Optim. 32(4), 1052-1067 (2016). https://doi.org/10. 1007/s10878-015-9908-4

37. B. Huang, J. Yu, X. Cheng, H. Chen, H. Liu, SINR based shortest link scheduling with oblivious power control in wireless networks. J. Netw. Comput. Appl. 77, 64-72 (2017). https://doi.org/10.1016/j.jnca.2016.10.012

38. X. Liu, M. Haenggi, Throughput analysis of fading sensor networks with regular and random topologies. EURASIP J. Wirel. Commun. Netw. 2005(4), 554-564 (2005). https://doi.org/10.1155/WCN.2005.554

39. J. Dams, M. Hoefer, T. Kesselheim, Scheduling in wireless networks with Rayleigh-fading interference. IEEE Trans. Mob. Comput. 14(7), 1503-1514 (2015). https://doi.org/10.1109/TMC.2014.2352278

40. J. Dams, M. Hoefer, T. Kesselheim, Scheduling in wireless networks with rayleigh-fading interference. In 24th ACM Symposium on Parallelism in Algorithms and Architectures, SPAA'12 (Pittsburgh, PA, USA, June 25-27, 2012), pp. 327-335. https://doi.org/10.1145/2312005.2312061

\section{Publisher's Note}

Springer Nature remains neutral with regard to jurisdictional claims in published maps and institutional affiliations. 